\title{
Crystallography of dispersed liquid crystalline phases studied by cryo-transmission electron microscopy
}

\author{
L. SAGALOWICZ, M. MICHEL, M. ADRIAN*, P. FROSSARD, \\ M. ROUVET, H. J. WATZKE, A. YAGHMUR †, L. DE CAMPO†, \\ O. GLATTER † \& M. E. LESER \\ Nestlé Research Center, Lausanne, Switzerland \\ *LAU, University of Lausanne, Switzerland \\ †Institute of Chemistry, University of Graz, Graz, Austria
}

Key words. Cryo-TEM, cubosomes, dispersion, freeze fracture, hexosomes, liquid crystals, mesophases, monoglyceride, SAXS, vesicles.

\section{Summary}

Low molecular weight surfactants, for example monoglycerides and phospholipids, form a multitude of self-assembled structures, such as inverted cubic or hexagonal mesophases, if brought into contact with water/oil. These mesophases can be dispersed in water using adequate surface-active materials such as low molecular weight surfactants or surface active polymers. In order to use such mesophase particles for incorporating drugs and aromas, it is essential to determine their internal crystallographic structure and to understand their mechanism of stabilization. Cryo-transmission electron microscopy was used to investigate the internal structure of different dispersed particles at various temperatures and oil contents. It is shown here that cryo-transmission electron microscopy, in combination with fast Fourier transform and tilting experiments, is effective in obtaining information on crystallographic structure, space group and morphology of particles with reversed bicontinuous cubic and hexagonal structures. In particular, using the presence or the absence of the $\{111\}$ reflections and viewing the same particle under different axes of observation allows one to discriminate between the Im $3 \mathrm{~m}$ and $\mathrm{Pn} 3 \mathrm{~m}$ space groups. A major advantage of cryotransmission electron microscopy is the ability to analyse single particles. This allows the identification of particles present at very low concentrations and the coexistence of particles with different internal self-assembly structures. With this technique we have obtained strong evidence for the presence of two cubic internal self-assembly structures with different space groups within the same dispersion. In addition, we

Correspondence to: Laurent Sagalowicz. Tel: +41 2178580 79; e-mail: Laurent.Sagalowicz@rdls.nestle.com found that cryo-transmission electron microscopy combined with tilting experiments enables the analysis of internal particle morphology, allowing the discussion of mechanisms for hexosome stabilization.

\section{Introduction}

Monoglycerides and phospholipids are known to form a multitude of self-assembled structures and liquid crystalline phases when put in contact with water (Larsson, 1989). These self-assembly structures have attracted significant attention in the literature. Applications, for example, are the solubilization and controlled release of various active molecules (Nylander et al., 1996; Caboi et al., 1997, 2001, 2002) such as nutrients (Shah et al., 2001) and flavours (Vauthey et al., 2000a,b). These liquid crystalline mesophases have also been observed in cells and are supposed to play an important role in biological mechanisms (Larsson, 1989).

The self-assembly structure formed depends not only on the type of surfactant used but also on many factors, such as temperature and water content (Lutton, 1965; Qiu \& Caffrey, 2000; Mezzenga et al., 2005). In the monoolein/water or monolinolein (MLO)/water system, a reversed microemulsion (also called a fluid isotropic or $\mathrm{L}_{2}$ phase), a lamellar $\left(\mathrm{L}_{\alpha}\right)$, a reversed hexagonal $\left(\mathrm{H}_{\mathrm{ii}}\right)$ and a reversed bicontinuous cubic liquid crystalline phase (Qiu \& Caffrey, 2000; de Campo et al., 2004) can form. Three different types of reversed bicontinuous cubic phases have been reported in the literature. At low hydration (or water content) a cubic assembly with a Ia3d space group is formed (gyroid type of infinite periodic minimal surface (IPMS), $\mathrm{C}_{\mathrm{G}}$ ), and at high hydration a cubic assembly with a Pn $3 m$ space group (double diamond type of infinite periodic minimal surface, $\mathrm{C}_{\mathrm{D}}$ ). In addition, the $\mathrm{Im} 3 \mathrm{~m}$ (primitive type of infinite periodic minimal surface, $C_{p}$ ) is observed when adding certain additives such as 
Poloxamer F127 (Landh, 1994; Gustafsson et al., 1996, 1997). The self-assembly structure is generally influenced by addition of an external molecule (Borné et al., 2000; Caboi et al., 2001, 2002). For example, the addition of an oil, such as tetradecane, induces a structural transition from cubic to $\mathrm{H}_{\mathrm{ii}}$ or $\mathrm{L}_{2}$ (Yaghmur et al., 2005) in the MLO/water system.

To enlarge the possible applications of self-assembly structures, the liquid crystalline phases (cubic or $\mathrm{H}_{\mathrm{ii}}$ ) have to be dispersed into an aqueous phase. In order to stabilize the obtained particles against coalescence or coagulation, an appropriate stabilizer, such as Pluronic F127, is used (Landh, 1994; Gustafsson et al., 1996, 1997; Larsson, 1999, 2000; Nakano et al. 2001, 2002; Spicer et al., 2001; de Campo et al., 2004). However, this stabilizer may change the internal structure, depending on the quantity used, of the dispersed particle. It has been observed that, when low concentrations of triblock copolymer (such as Pluronic F127) are added to the dispersed cubic phase formed in the system monoolein-water, the space group detected was Pn3m both in the dispersion and in the corresponding bulk phase in excess water, whereas in the presence of a higher amount of copolymer, the internal structure of the dispersed particle takes the space group Im3m (Gustafsson et al., 1996, 1997).

Recently, some of us reported on the influence of temperature (de Campo et al., 2004) and the addition of tetradecane (Yaghmur et al., 2005) to dispersed bicontinuous cubic particles made in the system MLO-water and stabilized by Pluronic F127. It was found that the internal particle structure is at each temperature identical to the structure formed in the nondispersed structure present in excess water. Upon heating or addition of tetradecane, the internal structure of the mesophase particles changes from reversed bicontinuous cubic via reversed hexagonal $\left(\mathrm{H}_{\mathrm{ii}}\right)$ to a $\mathrm{L}_{2}$-phase (de Campo et al., 2004; Yaghmur et al., 2005). Upon cooling, the opposite transformations are observed.

A major issue in the investigation of dispersed mesophase particles is the appropriate choice of analytical methods that allow structural characterization of the system. Different techniques such as small angle X-ray scattering (SAXS) (Gustafsson et al., 1997; Nakano et al., 2001), cryo-transmission electron microscopy (cryo-TEM) (Almgren et al., 2000), atomic force microscopy (Neto et al., 1999), dynamic light scattering and ${ }^{13}$ C NMR (Monduzzi et al., 2000; Nakano et al., 2001) have been applied. In the present study we made use of cryo-TEM to characterize the internal structure and shape of different dispersed particles. Cryo-TEM (Adrian et al., 1984; Dubochet et al., 1988; Talmon, 1996) is one of the best means of obtaining a direct image of the formed structure in a fluid. Cryo-TEM is nowadays a standard tool to study dispersed liquid crystalline phases (Andersson et al., 1995; Almgren et al., 1996; Gustafsson et al., 1996; Gustafsson et al., 1997; Almgren et al., 2000; Borné et al., 2001a,b, 2002; Spicer et al., 2001; de Campo et al., 2004; Yaghmur et al., 2005). However, in these previous studies, particles or particle types were viewed only along one crystallographic direction, which restricts the information obtained on the crystallographic structure, shape and defects. In particular with such limited information no space group can be identified.

A major advantage of cryo-TEM techniques lies in the possibility of analysing single particles. This allows the identification of coexisting particles with different internal self-assembly structures. Cryo-TEM is also extremely useful at very low particle concentrations, small particle sizes, and/or in complex systems for which other techniques such as small angle X-ray scattering (SAXS), NMR etc. will, in many circumstances, not lead to an identification of all particle types present.

In the present study, we show how cryo-TEM associated with tilting experiments can distinguish between a dispersed cubic and $\mathrm{H}_{\mathrm{ii}}$ phase particle (called respectively cubosome and hexosome) and how the space group of the reversed bicontinuous cubic phase particle can be identified. We use also cryo-TEM tilting experiments to identify morphology, shape and structural defects of dispersed hexosomes. Additionally, mechanisms for hexosome stabilization are proposed.

\section{Materials and methods}

\section{Materials}

MLO (emulsifierTS-PH039, glycerol monolinoleate) was supplied by Danisco A/S (Brabrand, Denmark). It consists of $93.8 \%$ monoglyceride and $4.1 \%$ diglycerides. The fatty acids are composed of $91.8 \%$ linoleate, $6.8 \%$ oleate and about $1 \%$ saturated fatty acids. Dimodan U was a gift from Danisco. The hydrocarbon tail is principally formed of: C18:2 (66\%), C18:1 (19\%), C18:0 ( $5 \%$ ) $\mathrm{C} 16: 0$ chains ( $8 \%$ ) and there was a residual amount of diglycerides (1.6\%). TS-T154 Diglycerol monooleate was supplied by Danisco. Tetradecane (TC) was obtained from Sigma Chemical Co. (St Louis, Missouri, U.S.A.). The stabilizer, Pluronic F127 $\left(\mathrm{PEO}_{99}-\mathrm{PPO}_{67}-\mathrm{PEO}_{99}\right)$, was a gift from the BASF Corporation (Mount Olive, New Jersey, U.S.A.). Emultop (partially hydrolysed lecithin that contains about 35\% lyso-lecithin and $65 \%$ lecithin) was from Degussa (Düsseldorf, Germany). These chemicals were used without further purification. MilliQ A10 (Millipore, St Quentin, France) water was systematically used.

\section{Formulation of aqueous dispersions}

The mixture of monoglycerides and oil was weighed and added to the aqueous stock solution of water plus stabilizer. Some samples containing all four ingredients were treated by ultrasonication for $20 \mathrm{~min}$ resulting in a milky dispersion. The typical composition of the $10 \mathrm{~g}$ prepared dispersions was $95 \mathrm{wt}-\%$ water and $5 \mathrm{wt}-\%$ of monoglycerides/oil/stabilizer mixture, whereby the total concentration of the stabilizer was $0.375 \mathrm{wt}-\%$. The particles are therefore in a large amount of excess water and far from the water separation line, which in the interior of the particles is about $40 \mathrm{wt}-\%$ for cubosomes (cubosomes ${ }^{\mathrm{TM}}$, Camurus, Sweden) and $20 \mathrm{wt}-\%$ for hexosomes (hexosomes ${ }^{\mathrm{TM}}$, Camurus, Sweden) (estimated from the corresponding bulk 
phases). Ultrasonication was carried out using a high intensity ultrasonic processor (SY-LAB G.m.b.H., Pukersdorf, Austria), at $30 \%$ of the maximum power, and $0.5 \mathrm{~s}$ pulses interrupted by $0.5 \mathrm{~s}$ breaks. No external sample cooling was applied. Some other samples were homogenized using a Polytron PT10-35 high shear mixer position 5 (Polytron, Luzern, Switzerland) for $5 \mathrm{~min}$ at $100 \mathrm{~g}$.

\section{Cryo-transmission electron microscopy}

A laboratory-built controlled environment vitrification system for cryo-TEM (very similar to the one described by Egelhaaf et al. (2000)) was used. Humidity in the environment chamber was close to $100 \%$ for all the dispersions. A droplet of $5 \mu \mathrm{L}$ of dispersion was deposited onto a 200 mesh copper grid (25 ${ }^{\circ} \mathrm{C}$, Quantifoil R2/2 or S7/2; both Jena, Germany) that was covered with a carbon film containing holes. It was left between two 595 filter papers (Schleicher \& Schuell, Dassel, Germany) for about $2 \mathrm{~s}$ before being propelled into liquid ethane. For some samples, a guillotine (without an environmental chamber) was used according to the procedure described by Sagalowicz et al. (2003). We conducted vitrification procedures at room temperature with and without the environmental chamber on the same sample, and the structure and morphology were identical for the two procedures. Frozen grids were stored in liquid nitrogen and transferred into a Cryo-holder (Gatan 626-DH, Pleasanton, CA, U.S.A.) that was kept at $-180^{\circ} \mathrm{C}$. Sample analysis was performed in a Philips CM12 TEM (Philips, Eindhoven, the Netherlands) at a voltage of $80 \mathrm{kV}$. Low dose procedures were applied to minimize beam damage. The images were recorded with a slow scan digital camera (Gatan 794) or onto negatives for high-resolution analysis.

\section{Results and discussion}

\section{Cubosomes}

Presence of vesicles in cubosome dispersions: direct imaging of the bilayers forming vesicle-like structures observed in cubosome dispersions Figure 1 shows a cryo-TEM image of a cubosome dispersion (made of Dimodan U and partially hydrolysed lecithin). Two types of objects are visible in this image: a vesicle with a complex shape and a cubosome. Note that for the vesicle and at the outside of the cubosome, two dark lines are visible coming from the polar heads of the surfactants (phospholipids and/or monoglycerides). The vesicle is consequently composed of a bilayer structure because one dark line represents one monolayer of the bilayer. The dimension of the bilayer (distance between the two dark lines) is approximately $4 \mathrm{~nm}$ which is closely related to the estimated distance between the polar heads of the two bilayer-forming monolayers (Tahara \& Fujiyoshi, 1994). The bilayer is observed using small defocus conditions (close to $900 \mathrm{~nm}$ ), so that the transfer function enhances distances around 3-4 nm.

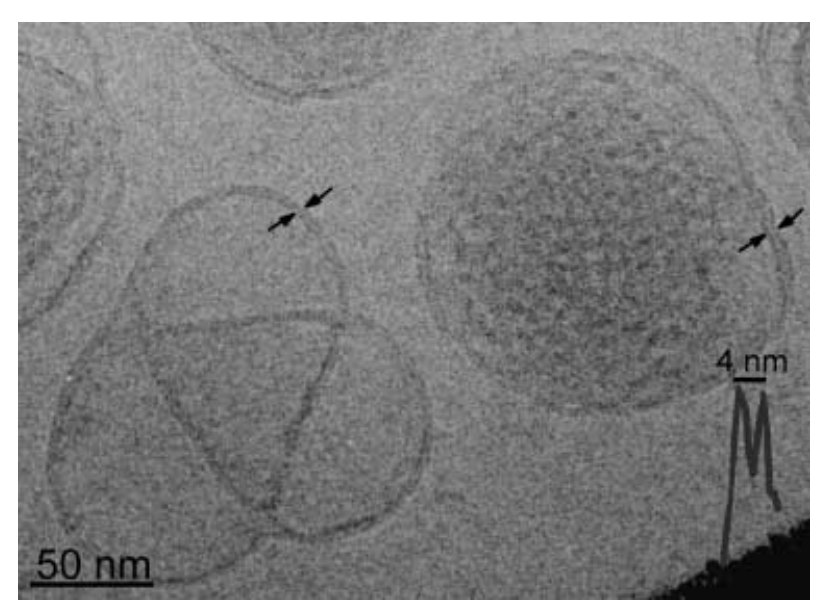

Fig. 1. Cryo-transmission electron microscopy image of a dispersion obtained by homogenization (Polytron) at $80{ }^{\circ} \mathrm{C}$, and that contains $4.5 \%$ Dimodan U and 0.5\% Emultop (composed of about 65\% lecithin and 35\% lyso-lecithin). Emultop and Dimodan U were first mixed before being introduced into water. Notice the presence of the double line associated with the lipidic bilayer. Overlay shows a line scan, of the intensity, of the double layer (arrows on the right of the cubosome).

\section{Crystallographic analysis of cubosomes}

Cubosomes with a space group Pn $3 m$ and with a lattice parameter of $8.5 \mathrm{~nm}$. Figure 2(a,b,d,f) shows cryo-TEM images of cubosomes of a dispersion made of MLO with Pluronic F127 as the stabilizer. The use of fast Fourier transform (FFT) analysis allows one to determine the structural symmetry inside the cubosome particles. FFT enables one to obtain very easily an optical diffractogram similar to an electron diffraction pattern. In this way periodic or repeatable distances present in the structure are detected right away, together with the symmetry of the motif even if these features are not very clearly observed in the direct image. Additionally, the distances and angles are much easier to determine using FFT than in the real image, because an average is more easily visualized in the FFT.

It can also be seen in Fig. 2(b) that the contrast is changing within the same particle and there can even be contrast inversion. However the FFT motif is still the same. Contrast of a relatively thick periodic object is complex in cryo-TEM as many unit cells are present in the thickness of the sample making direct interpretation of the image contrast difficult without simulation. It is known in material science that the contrast in the real image (lattice imaging) of crystals, like ceramics, metals or semiconductors, strongly depends on conditions, such as sample thickness, focus and precise orientation (Williams \& Carter, 1996).

In the cubosome particle image shown in Fig. 2(b), FFT analysis reveals a two dimensional (2D) hexagonal symmetry (Fig. 2c). It must be recalled that the projection of a 3D cubic array on 2D is hexagonal when visualized along the $<111>$ direction. In the cryo-TEM studies published so far on the characterization of the internal Pn3m cubic structure of 


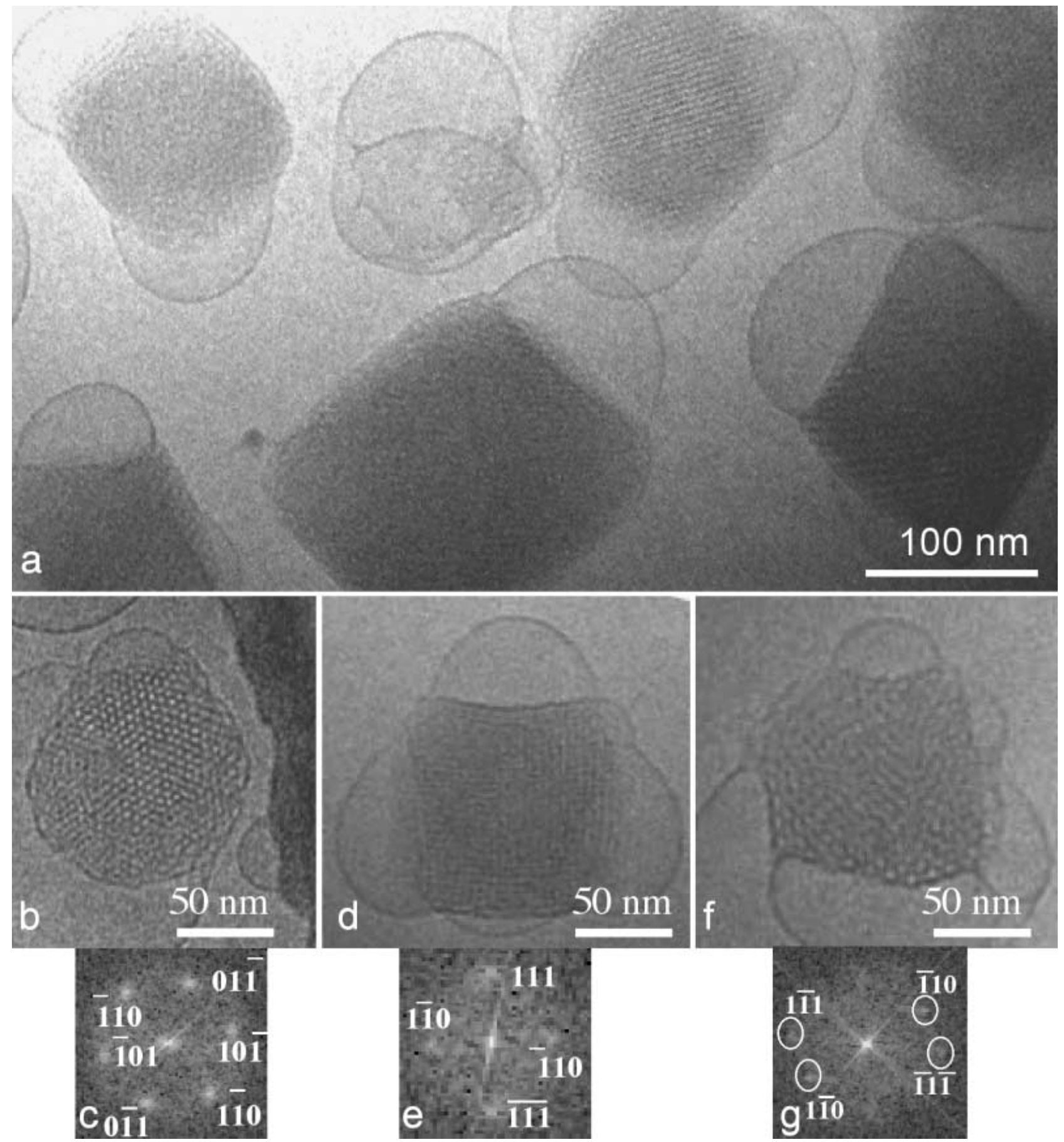

Fig. 2. Cryo-transmission electron microscopy image of a dispersion obtained by ultra-sonication and containing $4.625 \%$ monolinolein and $0.375 \%$ Pluronic F127. (a) General view. (b) The particle is orientated along the [111] direction. (c) Fourier transform of the particle in (b). (d) The particle is orientated along the [-1-12] direction. (e) Fourier transform of the particle in (d). (f) The particle is orientated close to the [110] direction. (g) Fourier transform of the particle in (f). The presence of the $\{111\}$ reflection demonstrates that the space group is Pn $3 \mathrm{~m}$ and not Im $3 \mathrm{~m}$. Parts (a)-(c) are reprinted with permission from de Campo et al. (2004). Copyright 2004 American Chemical Society.

monoglyceride based cubosomes, only 2D hexagonal symmetry has been observed (Gustafsson et al., 1997; Borné et al., 2001a,b, 2002). This does not enable one to fully determine the crystal structure and in particular to differentiate between the $\mathrm{H}_{\mathrm{ii}}$ structure and the cubic structure. However a closer look at other particles in the same sample also reveals the presence of other symmetries (Fig. 2d-g) corresponding to the $<112>$ and $<110>$ axis, demonstrating that the structure is cubic and not 
$\mathrm{H}_{\mathrm{ii}}$. As will be explained later, the only 2D symmetry which can be observed for the $\mathrm{H}_{\mathrm{ii}}$ structure is the hexagonal one.

With the information presented in Fig. 2, we can identify that the particle has an internal cubic structure as described above, as well as the space group or the type of cubic phase (gyroid, diamond or primitive). For this it is assumed that only the Pn $3 \mathrm{~m}$ (diamond) and $\mathrm{Im} 3 \mathrm{~m}$ (primitive) space groups are possible in cubosome dispersions because those are the only two space groups established in reversed bicontinuous cubic phases in excess water (Landh, 1994) or in reversed bicontinuous cubic phase dispersions (Gustafsson et al., 1997) in the system monoglyceride-Pluronic F127-water. The determination of the space group is performed as follows: in the FFT of the image viewed along the $<112>$ and $<110>$ axis, the $\{111\}$ reflection is present. The presence of the $\{111\}$ reflection demonstrates that the space group is Pn $3 m$ and not $\operatorname{Im} 3 m$ because for the space group $\operatorname{Im} 3 \mathrm{~m}$, the $\{111\}$ reflection is forbidden. The distance between the $\{110\}$ planes is $6.0 \mathrm{~nm}$ and the distance between the $\{111\}$ planes is close to $4.9 \mathrm{~nm}$ leading to a lattice parameter of $8.5 \mathrm{~nm}$ as also determined by SAXS (de Campo et al., 2004).

Tilting experiment with particles having the space group Pn $3 m$ Figure 3(a-b) shows a particle with a hexagonal symmetry where the $\{110\}$ type reflections contributes in the FFT. The electron beam is parallel to [111]. For this tilting experiment, it is crucial that one of the $\{110\}$ planes is normal to the axis of rotation of the TEM sample holder. In this case the $(-110)$ planes are normal to the axis of rotation of the sample holder. After rotating by $10^{\circ}$ the sample holder (not shown

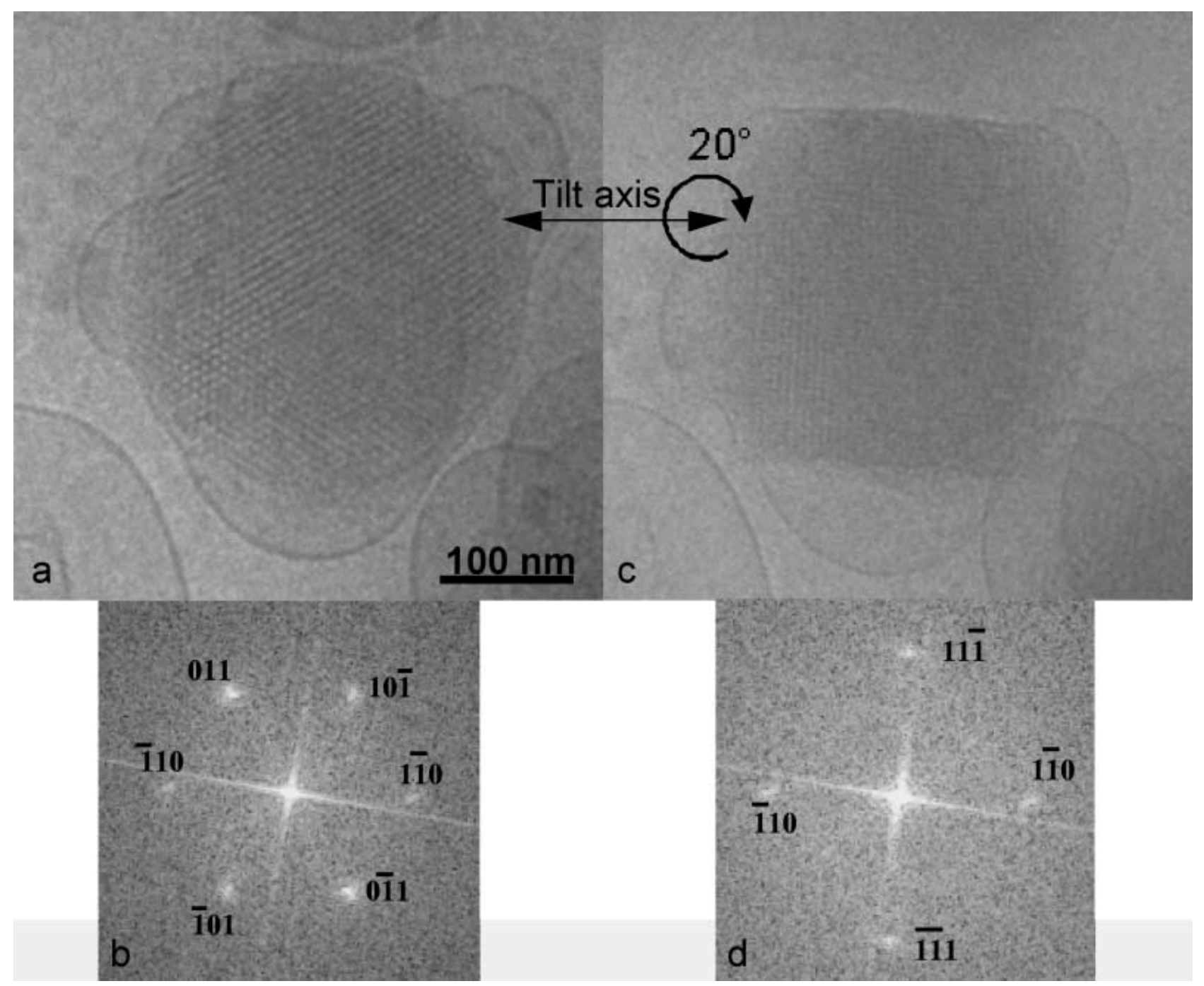

Fig. 3. Tilt series of a cubosome with the Pn $3 \mathrm{~m}$ space group. (a) Image at $0^{\circ}$ tilt. (b) Fast Fourier transform corresponding to (a). The particle is orientated along the [111] direction. (c) Image at $20^{\circ}$ tilt. (d) Fourier transform of (c) showing the $\{111\}$ reflection. The particle is orientated along the [112] direction. The dispersion has the same composition as the one used for Fig. 2. 
here), only the $(-110)$ planes are visible because the other $\{110\}$ planes are no longer parallel to the electron beam. More interesting, by rotating by $20^{\circ}$, the electron beam is parallel to the [112] direction and the (11-1) reflection contributes as shown in Fig. $3(\mathrm{c}-\mathrm{d}) .20^{\circ}$ is the angle between the [111] direction and the [112] direction, confirming that the particle is really observed along [112] in Fig. 3(c) and not along other directions such as the [100] that would correspond to a tilting angle of $55^{\circ}$. Now, as expected, after rotating by $30^{\circ}$, only the $(-110)$ and $(1-10)$ reflections are observed. The distance corresponding to the $(-110)$ reflection remains constant (about $6.3 \mathrm{~nm}$ ) during all the tilting series. The control of the exact orientation did not significantly influence the interplanar distances determined experimentally, at least when the periodic particle is relatively thick. Either the interplanar distance determined on the FFT remains approximately constant, or the reflection disappears (when the angle between the electron beam and the lattice plane is higher than about $5-10^{\circ}$ ). When the electron beam is not exactly aligned with the lattice plane, there is more noise in the image, and the uncertainty of distance determination is slightly higher. At higher tilt angles, the angle between lattice planes and electron beam is too high, and the planes are no longer observed.

With this approach, other space groups can be eliminated. For example, if one considers the gyroid Ia3d structure, the first allowed reflections are $\{211\},\{220\},\{321\}$ and $\{400\}$. The hexagonal motif (Fig. 3a-b) could only correspond to the [111] direction of observation. However, for the Ia $3 \mathrm{~d}$ space group, the $\{211\}$ and $\{220\}$ reflections should contribute when the electron beam is aligned with the [111] direction. Only one type of reflection is observed in this (or close to this) direction of observation, meaning that the $\{220\}$ (or the $\{211\})$ reflections are not present. In addition, for the Ia3d, there is no motif compatible with the one observed in Fig. 3(cd), which corresponds to a $20^{\circ}$ tilt from the [111] direction, even if one reflection is missing.

As mentioned earlier, in cryo-TEM, either the interplanar distance determined by the FFT analysis remains approximately constant, or the reflection disappears (when the periodic structure is thick enough). In other TEM techniques, the motif can be strongly influenced by the orientation. For example, in freeze fracture the surface is observed, and the sample can be tilted to almost any angle and a structure is still observed. However, the angle and distances between crystallographic features are strongly affected. Nonetheless, interpretation of fracture features can be used to discriminate between different space groups (Delacroix et al., 1993; Delacroix, 1998). For example, in the system formed by dioleoyl-phosphatidylcholinedioleoylglycerol, the analysis was compatible with the $\mathrm{Fd} 3 \mathrm{~m}$ space group but not with the $\mathrm{Fd} 3$ one.

Cubosome with a space group Im $3 m$ and a lattice parameter of $14 \mathrm{~nm}$ Figure 4(a) shows a cubosome observed along the $<100>$ direction and a square motif (Fig. 4a-b) is identified. The presence of a squared motif proves that a cubic structure is present as the $\mathrm{H}_{\mathrm{ii}}$ structure will not show this square motif. Hexagonal
Fig. 4. Cryo-transmission electron microscopy image of a dispersion corresponding to a lattice parameter of $14 \mathrm{~nm}$. (a) The particle is orientated along the [100] direction. (b) Fourier transform of the particle in (a). (c) The particle is orientated along the [111] direction. (d) Fourier transform of the particle in (c).

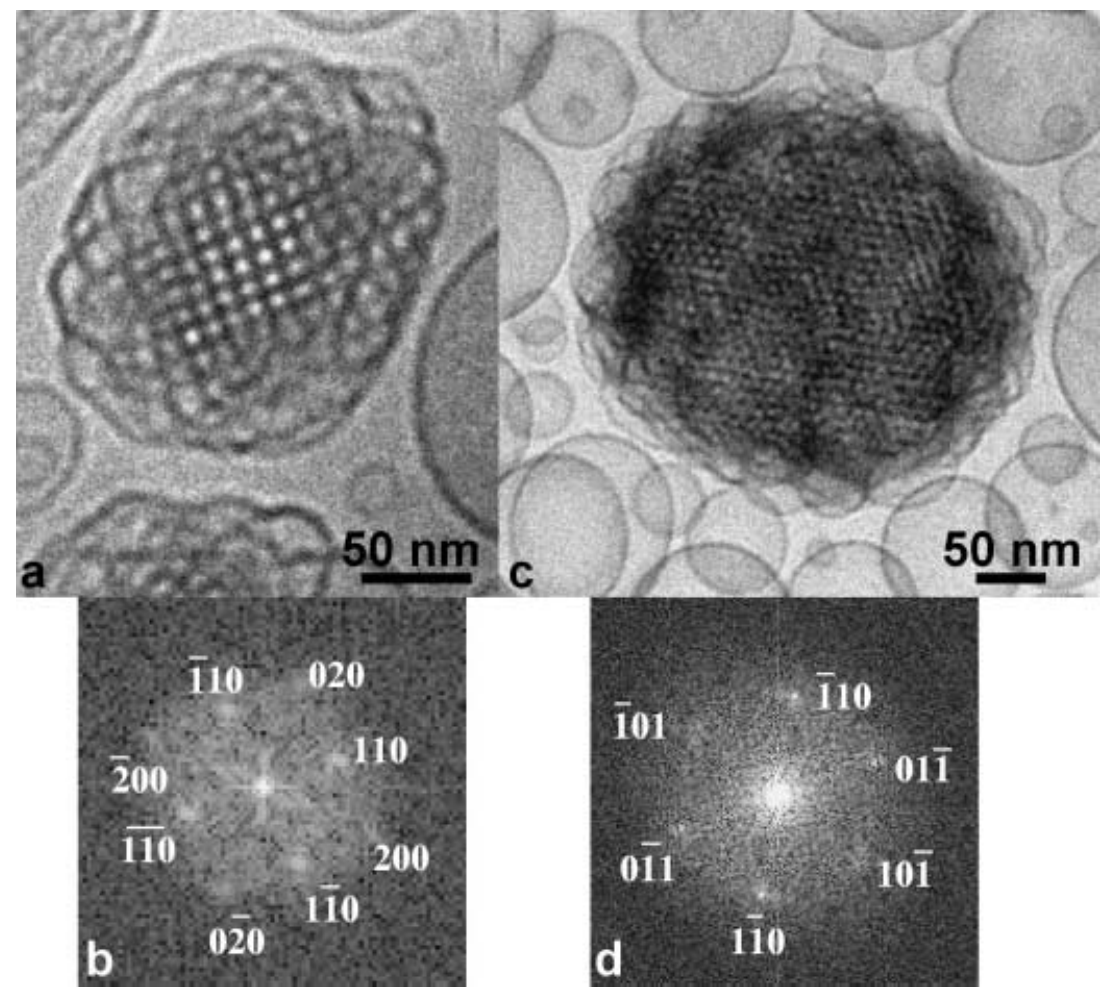




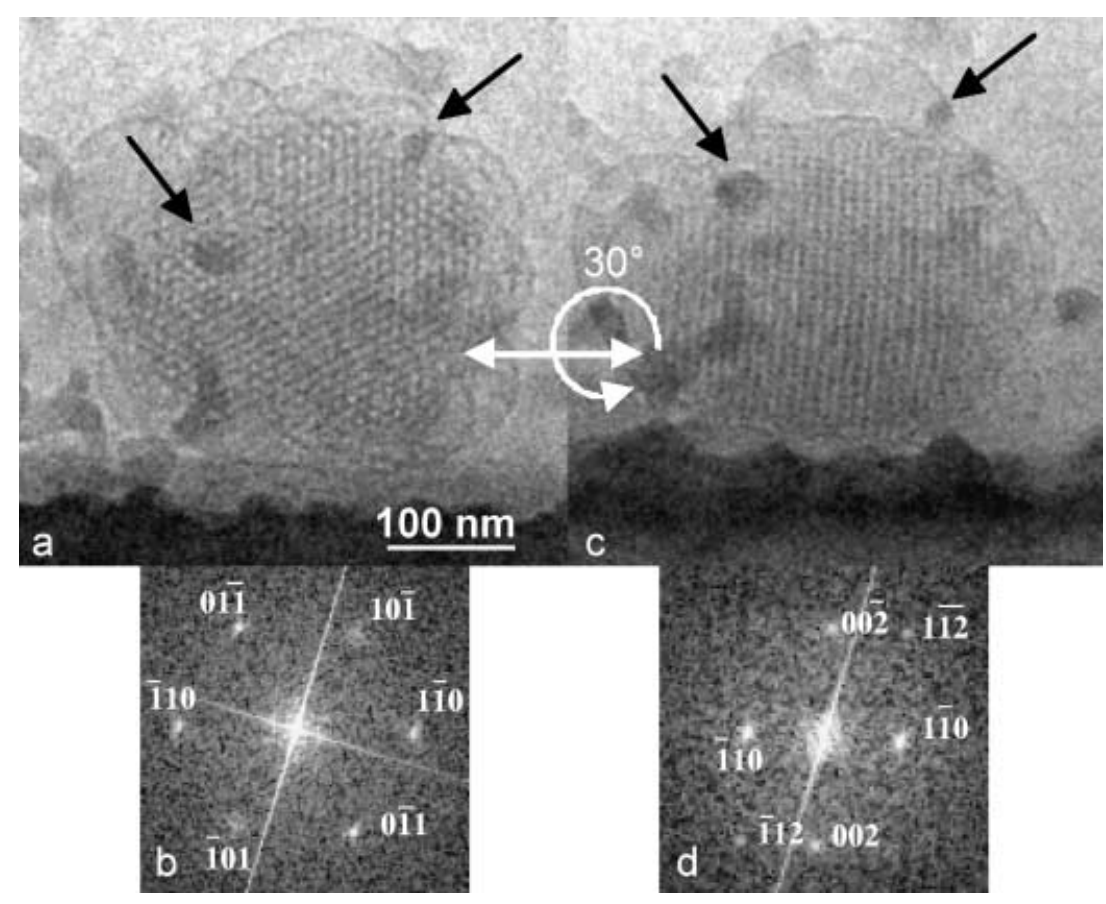

Fig. 5. Tilt series of a cubosome having the $\operatorname{Im} 3 \mathrm{~m}$ space group. (a) Image at $0^{\circ}$ tilt. (b) Fast Fourier transform corresponding to (a). The particle is orientated along the [111] direction. (c) Image at $30^{\circ}$ tilt. (d) Fourier transform of (c). The particle is orientated along the [110] direction. Note that the $\{002\}$ reflection is present but the $(1-11)$ and the (1-1-1) are absent, showing that the space group is $\operatorname{Im} 3 \mathrm{~m}$ and not Pn $3 \mathrm{~m}$. The composition of the dispersion used is $95 \mathrm{wt}-\%$ water, $3.7 \mathrm{wt}-\%$ monolinolein, 0.925 wt- $\%$ diglycerol monooleate and 0.375 wt- $\%$ Pluronic F127 and the dispersion was obtained by ultrasonication. Arrows indicate features which are probably surface contamination. motifs (Fig. 4c-d) are also observed. Notice that for this dispersion (Fig. 4), we never observed the $\{111\}$ reflection, which is a strong indication of the presence of $\mathrm{Im} 3 \mathrm{~m}$ symmetry. The $\{110\}$ spacing is about $10 \mathrm{~nm}$ leading to a lattice parameter of $14 \mathrm{~nm}$. In this work, it was found that particles having $\operatorname{Im} 3 \mathrm{~m}$ symmetry usually show a larger lattice parameter (in the range of $10-15 \mathrm{~nm}$ ) than particles having Pn3m symmetry (in the range of $6-12 \mathrm{~nm}$ ). The presence of particles observed along the [100] direction having a larger lattice parameter for the space group Im3m was previously reported by Gustafsson et al. (1997).

Tilting experiment with particles having the space group Im $3 m$ Figure 5(a-b) shows a particle having a hexagonal symmetry where the $\{110\}$ reflections contribute to the FFT. In this dispersion, the presence of particles with an Im $3 \mathrm{~m}$ space group was evidenced by SAXS. The electron beam is parallel to [111]. The (-110) planes are normal to the sample holder axis of rotation. After rotation by less than $20^{\circ}$, only the $(-110)$ planes are observed. However, by rotating the sample holder by about $30^{\circ}$, the (002) planes are also observed (Fig. 5c-d), which means that the electron beam is close to the [110] direction. The angle between [111] and [110] is about $35^{\circ}$, which is in good agreement with the $30^{\circ}$ tilt considering that the crystallographic plane can still be observed even if not exactly aligned with the electron beam. Note that the symmetry observed when the electron beam is aligned with the [110] is in full agreement with the Im $3 \mathrm{~m}$ space group but not with the Pn $3 m$ because the reflections (1-11) and (1-1-1) would have contributed for the latter. For the electron beam aligned with the [110] direction, focus series were used to check that the (1-11) and (1-1-1) reflections were really absent and their absence was not associated with a special focus and feature of the transfer function. The Ia3d (gyroid) reversed bicontinuous cubic structure can also very likely be discarded by crystallographic argument in a very similar way as was done when discussing the Pn3m structure. The hexagonal motif (Fig. 5a-b) could only correspond to the [111] direction of observation. However, for the Ia $3 d$ space group the $\{211\}$ and $\{220\}$ reflections should contribute when the electron beam is aligned with the [111] direction. Only one type of reflection is observed in this (or close to this) direction of observation meaning that the $\{220\}$ (or the $\{211\}$ ) reflections are not present. There could be confusion only if the $\{211\}$ reflections are absent (in the Ia3d) due to a zero form factor. However, SAXS on Ia3d structures made of unsaturated monoglycerides indicates that this reflection is strong. Therefore, from the cryo-TEM analysis, the presence of the reversed bicontinuous structure with a space group Ia $3 \mathrm{~d}$ is extremely unlikely. As mentioned in the Introduction, the presence of the $\mathrm{Im} 3 \mathrm{~m}$ space group in dispersions is due to the stabilizer, which is Pluronic F127. The $\mathrm{Im} 3 \mathrm{~m}$ space group is not observed in the binary phase diagram monoolein (or MLO)/water (Qiu \& Caffrey, 2000; de Campo et al., 2004). However, it is observed in bulk samples when adding stabilizers, such as Pluronic F127 (Land, 1994) or in dispersions containing the same stabililizers (Gustafsson et al., 1996, 1997). The Ia3d space group has not yet been observed in cubic phase dispersions of lipids (in this work and to the best of our knowledge in the literature), but it might be present with systems other than monoglycerides, or with the presence of additives. 
Evidence for a cubosome dispersion with two internal structures Figure 6 shows images obtained in a cubosome dispersion. As mentioned earlier, the only reported space group for reversed bicontinuous cubic phases are Pn $3 m$, Im $3 m$ and Ia 3d. We first consider only the space groups $\mathrm{Pn} 3 \mathrm{~m}$ and $\mathrm{Im} 3 \mathrm{~m}$ as these are the only ones yet reported for cubosome dispersions. The possible presence of the space group Ia $3 \mathrm{~d}$ will be discussed later. In Fig. 6(c, g), the $\{110\}$ reflection is visible because the $\{100\}$ reflection is absent for Pn $3 \mathrm{~m}$ and $\mathrm{Im} 3 \mathrm{~m}$ but the $\{110\}$ reflection is present. However, the corresponding planar distance is very different. In Fig. 6(c), it is about $7 \mathrm{~nm}$ (leading to a lattice parameter of $10 \mathrm{~nm})$, whereas in Fig. $6(\mathrm{~g})$ it is about $9.7 \mathrm{~nm}$ (leading to a lattice parameter of $13.5 \mathrm{~nm}$ ). In Fig. 6(g), the structure is clearly cubic because a squared motif is observed. For Fig. $6(\mathrm{~d}-\mathrm{e})$, the $\{110\}$ reflection is observed that corresponds to the $7 \mathrm{~nm}$ distance, also observed in Fig. 6(b-c). Additionally, in the same particle (Fig. 6d) the $\{111\}$ reflection is observed (Fig. 6e) giving strong evidence that a cubic structure with a Pn3m space group is formed. It can be concluded that in this sample two cubic phases coexist within the same dispersion: one with a space group Pn $3 \mathrm{~m}$ and a lattice parameter of $10 \mathrm{~nm}$ and another with a lattice parameter of $13.5 \mathrm{~nm}$. It is likely that the cubic phase with the larger lattice parameter has a space group Im $3 \mathrm{~m}$ because it has a larger lattice parameter and is observed along the $<100>$ direction, which is characteristic of the Im3m space group. The ratio between the lattice parameter of the two structures is qualitatively in good agreement with the theoretical value obtained from the Bonnet transformation (1.27) between cubic phases having the space group Pn3m and Im3m (Hyde, 1996). In other studies, the ratio between the two lattice parameters has also been found to be slightly higher than 1.27 (Abraham et al., 2005). The presence of the Ia $3 \mathrm{~d}$ space group is also unlikely in this dispersion. As explained earlier, Fig. 6(b-e) shows that the corresponding particles (lattice parameter of $10 \mathrm{~nm}$ ) have not the space group Ia $3 d$ but $\operatorname{Pn} 3 m$ as the $\{211\}$ reflection is not observed and the $\{111\}$ reflection is. The Bonnet relation also strongly suggests that the other types of particles (lattice parameter of $13.5 \mathrm{~nm}$ ) have not the space group Ia3d but Im3m. The number of particles having a large lattice parameter $(13.5 \mathrm{~nm})$ is much lower than the number of particles having a lattice parameter of $10 \mathrm{~nm}$. Although SAXS is a very good method to determine the crystallographic structure fully, it is probably not sensitive enough to detect such a low concentration of small particles. On top of that, when cubosomes are present in the dispersion, the simultaneous presence of vesicles in the dispersion cannot be detected by SAXS due to a comparatively too weak signal.

\section{Hexosomes}

The formation of hexosomes, i.e. particles having a reversed hexagonal internal structure, is an alternative to cubosome particle formation. The addition of a certain amount of tetradecane transforms cubosomes into hexosomes (Yaghmur et al., 2005). The particles show internal arrangements of a hexagonal symmetry and/or curved striations (Fig. 7). No or only a little vesicle formation and no other symmetry is detectable in the hexosome dispersion. The presence of a 2D hexagonal symmetry is obviously good evidence for the presence of a $\mathrm{H}_{\mathrm{ii}}$ mesophase structure. However, this is not complete proof because cubosomes may also have this 2D hexagonal symmetry. In particular, a large number of cubosomes having the space group Pn3m show a 2D hexagonal arrangement by cryo-TEM. However, by contrast to cubosomes, when looking at hexosomes, no other 2D symmetry than the hexagonal one is observed. All the particles show a hexagonal internal arrangement or parallel lines (which may be curved).

As the observed hexagonal tubes in the hexosome particles are infinitely long (or limited by the particle size), there is no periodicity in the direction of the cylindrical tube, explaining why only the hexagonal arrangement or parallel lines are observed by cryo-TEM. Another striking feature is the presence of curved striations (Fig. $7 \mathrm{~b}-\mathrm{c}$ ) that are also observed in hexosome dispersions described in the literature (Almgren et al., 1996; Gustafsson et al., 1997; de Campo et al., 2004; Yaghmur et al., 2005). They have also been observed in the bulk $H_{\text {ii }}$ structure (Siegel et al., 1989; Frederik et al., 1991). Figure 7(b) could suggest the presence of a multilamellar or onion-type vesicle or liposome. However, the particles show both curved striations and $\mathrm{H}_{\mathrm{ii}}$ symmetry (Fig. 7a). The particle shown in Fig. 8(a), shows a $\mathrm{H}_{\mathrm{ii}}$ symmetry. FFT leads to a lattice parameter of $6.5 \mathrm{~nm}$, which is in good agreement with the lattice parameter found by SAXS (Yaghmur et al., 2005). If the particle imaged in Fig. 8(a) is rotated by $40^{\circ}$, curved striations become visible (Fig. 8b). This strongly indicates that the same feature is associated with both hexagonal symmetry in 2D and curved striations. The closed packed tubes forming the $\mathrm{H}_{\mathrm{i}}$ structure are not very rigid and, consequently, can deform. Bending of the tubes was also observed in earlier work (Siegel et al., 1989; Frederik et al., 1991). The effect of tilt on hexosome images is quite different from that of cubosomes. If cubosomes are tilted by more than $15^{\circ}$, the motif initially observed generally disappears. For hexosomes, the hexagonal motif is still present in some regions after $40^{\circ}$ of tilting. This comes from the fact that hexosomes are not really single crystals because the longitudinal axis of the cylinders is bent and therefore, it is more likely that some crystallographic planes are parallel to the electron beam after a very large tilt.

The presence of curved tubes in the hexosome particles is probably associated with the stabilization mechanism of hexosomes. Hexosome stabilization requires that two different surfaces are stabilized. One surface is at the outside of the cylinder tubes (Fig. 9, left part) which is completely lipophilic and easily stabilized by a surfactant layer, and the second surface is the end of the tube (Fig. 9, left part) where both the hydrophilic and lipophilic parts of the monoglyceride molecules 


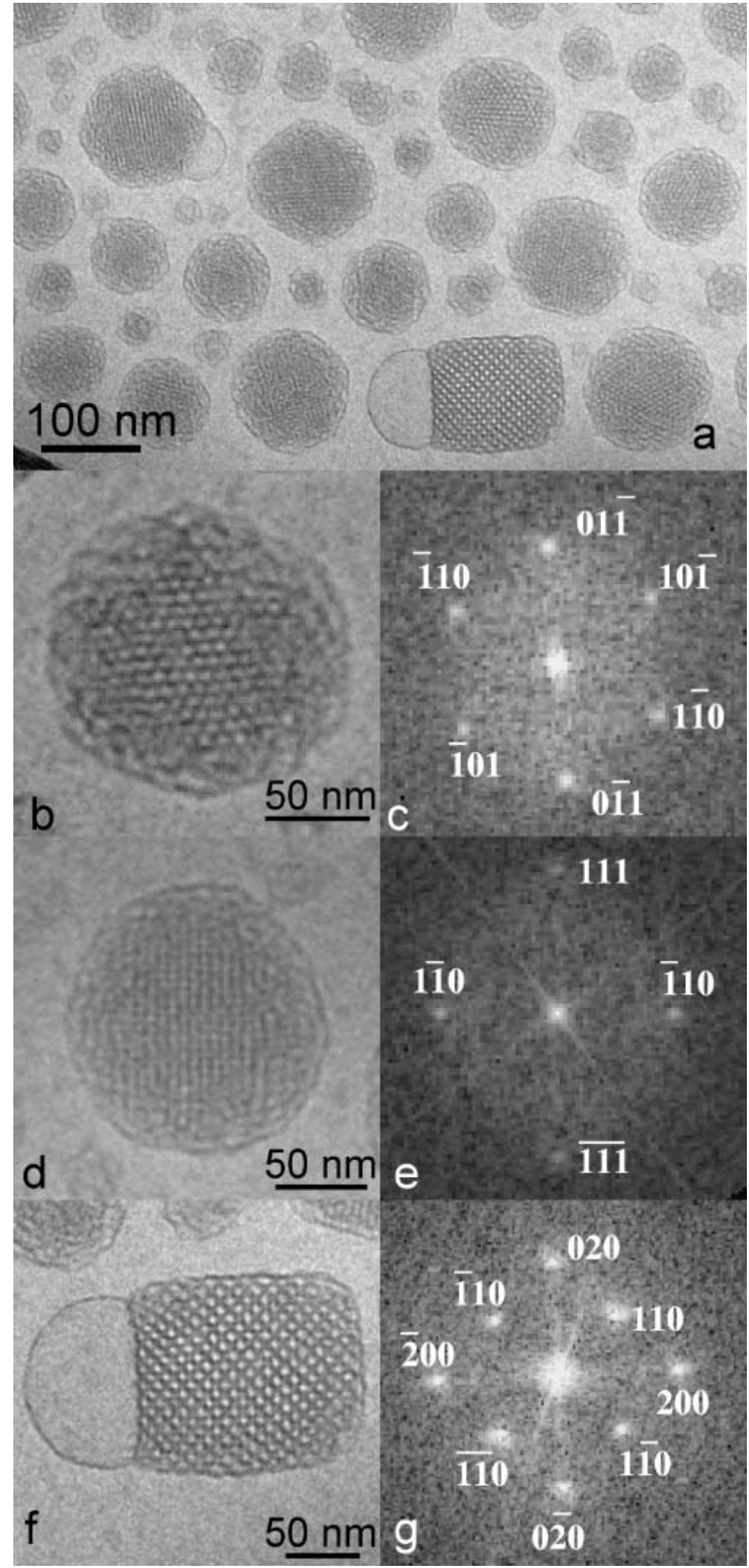

Fig. 6. Cryo-transmission electron microscopy image of a dispersion containing particles with two different internal structures. (a) General image. (b) Image of a particle with the space group Pn $3 \mathrm{~m}$. (c) Fourier transform of the particle in (b). (d) Image of a particle with the space group Pn3m and viewed along the [11-2] direction where the $\{111\}$ reflection is present, demonstrating that the space group is Pn $3 \mathrm{~m}$. (e) Fourier transform of the particle in (d). (f) Image of a particle likely with the space group Im3m. (g) Fourier transform of the particle in (f). The composition of the dispersion used is $95 \mathrm{wt}$ \% water, 4 wt-\% Dimodan U and 1 wt- $\%$ sodium caseinate and the dispersion was obtained by homogenization (Polytron) at $80{ }^{\circ} \mathrm{C}$. 
Fig. 7. Cryo-transmission electron microscopy image of hexosome dispersions. (a) Dispersion obtained by ultrasonication with $0.74 \%$ tetradecane and 3.89 wt-\% monolinolein and 0.3625 wt-\% Pluronic F127. Notice the presence of particles with hexagonal motif and/or curved striations. Reprinted with permission from Yaghmur et al. (2005). Copyright 2005 American Chemical Society. (b) Particle in a dispersion obtained with 4.625 wt-\% monolinolein, 0.375 wt-\% Pluronic F127 and vitrification at a dispersion temperature of $55{ }^{\circ} \mathrm{C}$. Reprinted with permission from de Campo et al. (2004). Copyright 2004 American Chemical Society. (c) Particle in the same dispersion as in (b) and vitrification obtained at a dispersion temperature of $50{ }^{\circ} \mathrm{C}$.
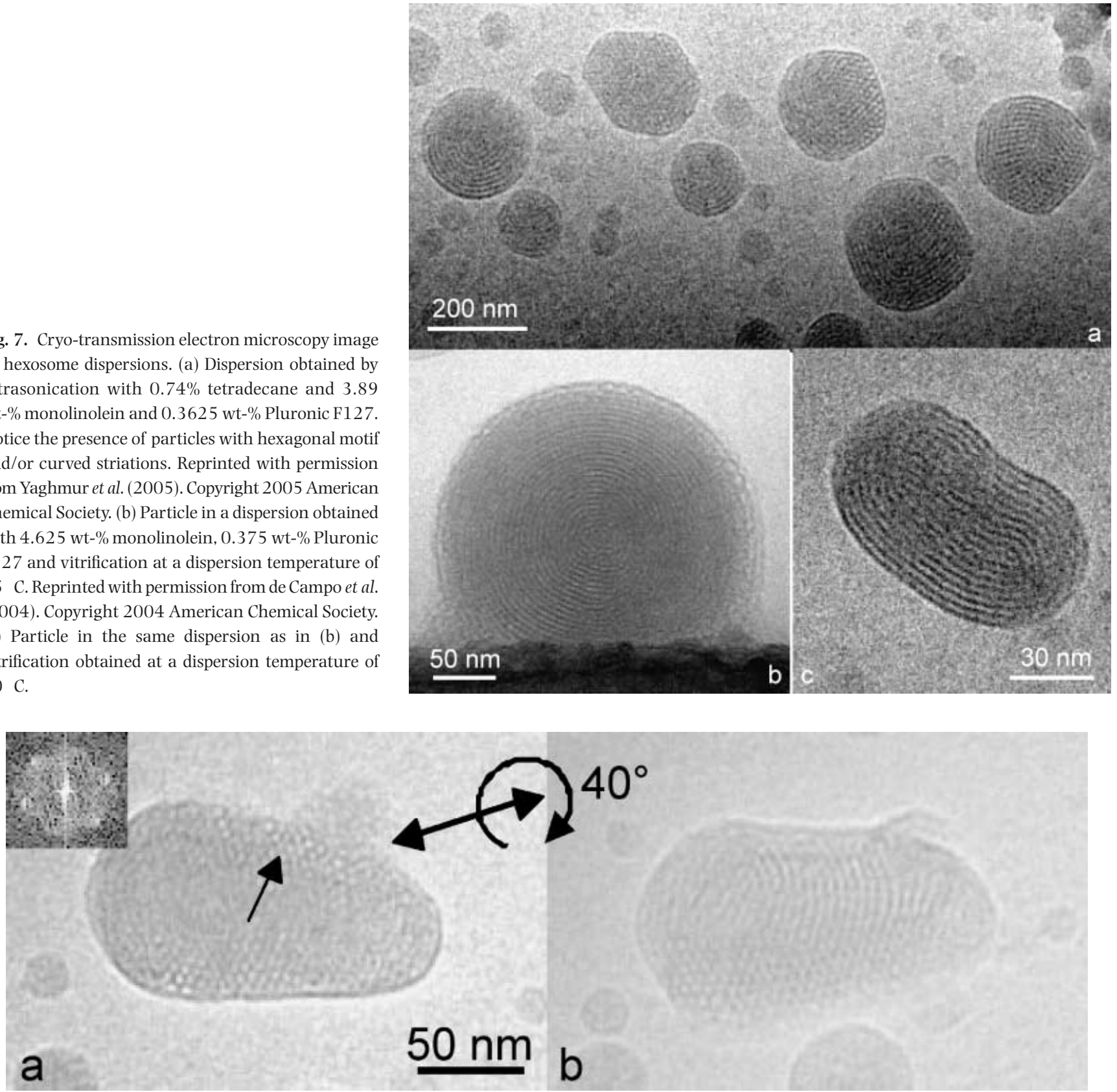

Fig. 8. Cryo-transmission electron microscopy image of the same hexosome viewed under two directions. (a) The hexagonal motif is visible, inset is the fast Fourier transform (FFT) (taken from the arrowed region) showing the hexagonal arrangement. (b) Same particle as (a) but rotated by $40^{\circ}$. Curved striations are present where the hexagonal motif was and in the region where the FFT (inset of (a)) was obtained (arrowed region). The dispersion was the same as that used for Fig. 7(a).

are in contact with water, if not stabilized. For example, if we imagine that a spherical hexosome is made up of straight tubes, many tube endings need to be closed. As depicted in the sketch in Fig. 9 (right part), the tubes can become circular and ideally can be closed by each other in such a way that no tube endings are present any more. This can also be achieved by folding one single tube. The curved tube(s) are closed and packed in a hexagonal array as it is the case for the original $\mathrm{H}_{\mathrm{ii}}$ structure. The hexagonal array is normal to the tube axis and the 2D structure has a hexagonal symmetry. The surface of such hexosome particles is completely lipophilic and thought to be easily stabilized, mainly by the adsorption of the stabilizer in a similar way as oil droplets are stabilized by the adsorption of surfactants on the oil droplet surface.

In the middle of a hexosome particle, i.e. less than about $20 \mathrm{~nm}$ away from the particle centre, no regular curved striations 


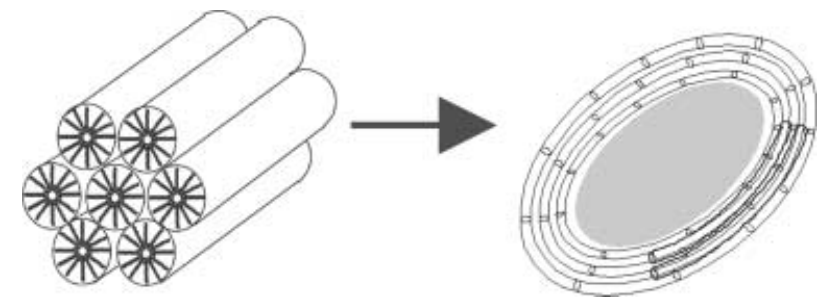

Fig. 9. Schematic of a non stabilized hexosome is shown on the left, stabilization achieved by turning the long tubes is shown on the right. The stabilizer covers the whole particle.

can be observed in the cryo-TEM images. In this case, assuming the presence of similar circular tubes as observed at the outside of the hexosome, the tubes have a high energy as their curvature is large. Either the tubes are curved and closed but in a different manner with a more energetically favourable configuration, or the tubes are closed by a half reversed micellar cap, as explained below.

Some particles (see for example fig. 5b in Yaghmur et al., 2005) have still a hexagonal shape, suggesting that the longitudinal axis of the tubes forming the $\mathrm{H}_{\mathrm{ii}}$ structure remains straight. Only close to the surface can the tube bend and turn by $180^{\circ}$ to close on to another straight tube. Half of a reversed micelle may also be present at the end of a tube to avoid contact of the lipophilic part of the monoglyceride with water. Such a mechanism of stabilization is also possible, especially when the composition is close to the limit of the appearance of the reversed microemulsion phase.

There are still other driving forces to explain the presence of curved striations. For example, if after the dispersion process, long and thin bundles of hexagonally packed cylinders are formed, their stabilization will require a large amount of surfactants and will correspond to a high interfacial area and therefore to a high energy. It might be more favourable to bend the cylinders like a horseshoe to reduce the interfacial area, explaining why truncated circles are also observed.

\section{Conclusions}

In this work we have shown that cryo-TEM associated with the use of tilting series and FFT is a reliable and effective method for elucidation of the internal structure of particles with a reversed cubic or reversed hexagonal. In particular, it is possible to discriminate between particles with space groups $\mathrm{Pn} 3 \mathrm{~m}$ and $\mathrm{Im} 3 \mathrm{~m}$ because the $\{111\}$ reflection is observed for Pn $3 \mathrm{~m}$ but is absent for $\mathrm{Im} 3 \mathrm{~m}$. In addition, a model for hexosome stabilization has been proposed.

\section{Acknowledgements}

We warmly thank Fred Chavannes, Olivier Girault, JeanAlbert Stucki, Dino Tomasi and Laurent Peissard (Nestlé
Research Center, Lausanne) for building the environmental chamber for the cryo-TEM analysis. Drs E. Hughes, K. Moffit and E. Prior are greatly acknowledged for reading the manuscript.

\section{References}

Abraham, T., Hato, M. \& Hirai, M. (2005) Polymer-dispersed bicontinuous cubic glycolipid nanoparticles. Biotechnol. Prog. 21 (1), 255-262.

Adrian, M., Dubochet, J., Lepault, J. \& McDowall, A.W. (1984) Cryoelectron microscopy of viruses. Nature, 308, 32-36.

Almgren, M., Edwards, K. \& Gustafsson, J. (1996) Cryotransmission electron microscopy of thin vitrified samples. Curr. Opin. Coll. Interface Sci. 1 (2), 270-278.

Almgren, M., Edwards, K. \& Karlsson, G. (2000) Cryo transmission electron microscopy of liposomes and related structures. Coll. Surf., A: Physicochem. Eng. Asp. 174 (1-2), 3-21.

Andersson, S., Jacob, M. \& lidin, S. (1995) Structure of cubosome - a closed lipid bilayer aggregate. Z. Kristallographie, 210, 315-318.

Borné, J., Nylander, T. \& Khan, A. (2000) Microscopy, SAXD, and NMR studies of phase behavior of the monoolein-diolein-water system. Langmuir, 16 (26), 10044-10054.

Borné, J., Nylander, T. \& Khan, A. (2001a) Phase behavior and aggregate formation for the aqueous monoolein system mixed with sodium oleate and oleic Acid. Langmuir, 17 (25), 7742-7751.

Borné, J., Nylander, T. \& Khan, A. (2001b) Vesicle formation and other structures in aqueous dispersions of monoolein and sodium oleate. J. Colloid Interface Sci. 257 (2), 310-320.

Borné, J., Nylander, T. \& Khan, A. (2002) Effect of Lipase on MonooleinBased Cubic Phase Dispersion (Cubosomes) and Vesicles. J. Phys. Chem. B, 106 (40), 10492-10500.

Caboi, F., Amico, G.S., Pitzalis, P., Monduzzi, M., Nylander, T. \& Larsson, K. (2001) Addition of hydrophilic and lipophilic compounds of biological relevance to the monoolein/water system. I. Phase behavior. Chem. Phys. Lipids, 109 (1), 47-62.

Caboi, F., Borné, J., Nylander, T., Khan, A., Svendsen, A. \& Patkar, S. (2002) Lipase action on a monoolein/sodium oleate aqueous cubic liquid crystalline phase-a NMR and X-ray diffraction study. Coll. Surf., B: Biointerfaces, 26 (1-2), 159-171.

Caboi, F., Nylander, T., Razumas, V., Talaikyte, Z., Monduzzi, M. \& Larsson, K. (1997) Structural effects, mobility, and redox behavior of vitamin $\mathrm{K} 1$ hosted in the monoolein/water liquid crystalline phases. Langmuir, 13 (20), 5476-5483.

de Campo, L., Yaghmur, A., Sagalowicz, L., Leser, M.E., Watzke, H. \& Glatter, O. (2004) Reversible phase transitions in emulsified nanostructured lipid systems. Langmuir, 20 (13), 5254-5261.

Delacroix, H. (1998) Crystallographic analysis of freeze-fracture electron micrographs: application to the structure determination of cubic lipid-water phases. J. Microsc. 192 (3), 280-292.

Delacroix, H., Gulik-Krzywicki, T., Mariani, P. \& Luzzati, V. (1993) Freeze fracture electron microscope study of lipid systems. J. Mol. Biol. 229, 526-539.

Dubochet, J., Adrian, M., Chang, J., Homo, J.-C., Lepault, J., McDowall, A.W. \& Schultz, P. (1988) Cryo-electron microscopy of vitrified specimens. Quart. Rev. Biophys. 21 (2), 129-228.

Egelhaaf, S.U., Schurtenberger, P. \& Müller, M. (2000) New controlled environment vitrification system for cryo-transmission electron microscopy: design and application to surfactant solution. J. Microsc. 200 (2), 128-139. 
Frederik, P.M., Burger, K.N.J., Stuart, M.C.A. \& V.A. (1991) Lipid polymorphism as observed by cryo-electron microscopy. Biochim. Biophys. Acta, 1062, 133-141.

Gustafsson, J., Ljusberg-Wahren, H., Almgren, M. \& Larsson, K. (1996) Cubic lipid-water phase dispersed into submicron particles. Langmuir, 12 (20), 4611-4613.

Gustafsson, J., Ljusberg-Wahren, H., Almgren, M. \& Larsson, K. (1997) Submicron particles of reversed lipid phases in water stabilized by a nonionic amphiphilic polymer. Langmuir, 13 (26), 6964-6971.

Hyde, S.T. (1996) Bicontinuous structures in lyotropic liquid crystals and crystalline hyperbolic surfaces. Curr. Opin. Solid State Mat. Sci. 1 (5), 653-662.

Landh, T. (1994) Phase Behavior in the System Pine Needle Oil Monoglycerides-Poloxamer 407-Water at 20. J. Phys Chem. 98 (34), 8453-8467.

Larsson, K. (1989) Cubic lipid-water phase. Structure and biomembrane aspects. J. Phys. Chem. 93 (21), 7304.

Larsson, K. (1999) Colloidal dispersions of ordered lipid-water phases. J. Dispers. Sci. Technol. 20 (1-2), 27-34.

Larsson, K. (2000) Aqueous dispersions of cubic lipid-water phases. Curr. Opin. Col. Interf. Sci. 5 (1-2), 64-69.

Lutton, E.S. (1965) Phase behavior of aqueous systems of monoglycerides. J. Am. Oil Chem. Soc., 42 (12), 1068-1070.

Mezzenga, R., Meyer, C., Servais, C., Romoscanu, A., Sagalowicz, L. \& Hayward, R. (2005) Shear rheology of lyotropic liqid crystals: a case study. Langmuir, 21 (8), 3322-3333.

Monduzzi, M., Ljusberg-Wahren, H. \& Larsson, H. (2000) A 13CNMR study of aqueous dispersions of reversed lipid phases. Langmuir, 16, 7355-7358.

Nakano, M., Sugita, A., Matsuoka, H. \& Handa, T. (2001) Small-angle Xray scattering and 13C NMR investigation on the internal structure of 'cubosomes'. Langmuir, 17 (13), 3917-3922.

Nakano, M., Teshigawara, T., Sugita, A. et al. (2002) Dispersions of liquid crystalline phases of the monoolein/oleic acid/Pluronic F127 system. Langmuir, 18 (24), 9283-9288.

Neto, C., Aloisi, G., Baglioni, P. \& Larson, K. (1999) Imaging soft matter with the atomic force microscope: cubosomes and hexosomes. Physical Chemistry B, 103, 3896-3899.
Nylander, T., Mattisson, C., Razumas, V., Miezis, Y. \& H. (1996) A study of entrapped enzyme stability and substrate diffusion in a monoglyceridebased cubic liquid crystalline phase. Coll. Surf., A: Physicochem. Eng. Asp. 114, 311-320.

Qiu, H. \& Caffrey, M. (2000) The phase diagram of the monolein/water system: metastability and equilibrium aspects. Biomaterials, 21, 223234.

Sagalowicz, L., Michel, M., Watzke, H.J. \& Adrian, M. (2003) Advanced electron microscopy methods to characterise food colloids. Science Technology and Education of Microscopy: an overview (ed. by A. Méndez-Vilas) 557. Formatex, Extremadura.

Shah, J.C., Sadhale, Y. \& Dakshina, M.C. (2001) Cubic phase gels as drug delivery systems. Adv. Drug Deliv. Rev. 47, 229-250.

Siegel, D.P., Burns, J.L.H., C.M. \& Talmon, Y. (1989) Intermediates in membrane fusion and bilayer/nonbilayer phase transitions imaged by time-resolved cryo-transmission electron microscopy. Biophys. J. 56, $161-169$.

Spicer, P.T., Hayden, K.L., Lynch, M.L., Ofori-Boateng, A. \& Burns, J.L. (2001) Novel process for producing cubic liquid crystalline nanoparticles (cubosomes). Langmuir, 17 (19), 5748-5756.

Tahara, Y. \& Fujiyoshi, Y. (1994) A new method to measure bilayer thickness: Cryo-electron microscopy of frozen hydrated liposomes and image simulation. Micron, 25, 141.

Talmon, Y. (1996) Transmission electron microscopy of complex fluids: the state of the art. Ber. Bunsenges. Phy. Chem. 100 (3), 364-372.

Vauthey, S., Milo, C., Frossard, P., Garti, N., Leser, M.E. \& Watzke, H.J. (2000a) Structured fluids as microreactors for flavor formation by the Maillard reaction. J. Agric. Food Chem. 48 (10), 4808-4816.

Vauthey, S., Visani, P., Frossard, P., Garti, N., Leser, M.E. \& Watzke, H.J. (2000b) Release of volatiles from cubic phases monitoring by gas sensors. J. Dispers. Sci. Technol. 21 (3), 263-278.

Williams, D.W. \& Carter, B. (1996) Transmission Electron Microscopy: a Textbook for Materials Science. Plenum Press, New York.

Yaghmur, A., de Campo, L., Sagalowicz, L., Leser, M.E. \& Glatter, O. (2005) Emulsified microemulsions and oil-containing liquid crystalline phases. Langmuir, 21, 569-577. 